\title{
Assessment of the usefulness of pipelle biopsy in gynecological diagnostics
}

\author{
Szymon Piątek, Grzegorz Panek, Mirosław Wielgoś \\ $1^{\text {st }}$ Department of Obstetrics and Gynecology, Medical University of Warsaw, Poland
}

\begin{abstract}
Objectives: The aim of this study is to assess the effectiveness of pipelle in sampling diagnostic material from the uterine cavity and determining clinical factors, which may affect its effectiveness.

Material and methods: The retrospective analysis included 312 patients who underwent pipelle biopsy because of various indications. Evaluated factors which may affect the effectiveness of this method were: types of indications for the procedure, age, BMI, anteflexion or retroflexion of the uterus, presence of uterine fibroids, miscarriages, natural deliveries, deliveries in general.

Results: In 259 (83.01\%) cases sampled material allowed for a histopathological diagnosis. In 53 (16.99\%) of the women we failed to sample tissue material from the uterine cavity. Indications for the procedure, BMI, age and menopausal status were factors of potential impact on the diagnostic effectiveness $(p<0.05)$. The diagnostic value of pipelle was found to be the lowest in women with overweight and obesity (BMI > 28), post-menopausal women, women over 55 years of age and where biopsy was performed for indications other than abnormal uterine bleeding. On the other hand, pipelle shows clearly the highest effectiveness in sampling diagnostic material from the uterine cavity in young women ( $<45$ years of age), menstruating women, women with normal body weight or underweight $(\mathrm{BMI}<23)$ and abnormal uterine bleeding. Conclusions: Choosing a pipelle as an endometrial biopsy method should consider the indications, BMI, age and menopausal status. Omission of these factors can increase non-diagnostic outcomes resulting in necessity of repeated biopsies, the risk of complications and increases the costs of health care system.
\end{abstract}

Key words: dilatation and curettage, pipelle, endometrial biopsy

\section{INTRODUCTION}

Biopsy of the endometrium is the principal method used to detect pathological changes in the uterine cavity. Diagnosis depends on obtaining adequate tissue material necessary for microscopic analysis. In everyday practice none of the methods used guarantees sampling an adequate quantity of tissue. What should be taken into account in the choice of the diagnostic method is its sensitivity and specificity, easiness of performance, availability and acceptance by patients. The economic aspect cannot be neglected either. Absence of the need for general anaesthesia and a possibility of performing the procedure on an outpatient basis lower the cost, which is of importance in the economic climate of today.
Curettage of the uterine cavity is considered to be a method of choice in numerous clinical situations [1]. Yet, even this examination is no guarantee of obtaining diagnostic material from the uterine cavity and thus giving a diagnosis [2]. It has been estimated that abrasion allows for the inspection of $50-60 \%$ of the uterine cavity surface while the percentage of non-diagnostic results reaches 3\% [3-5]. This method, requiring general anaesthesia and hospitalization, entails a high risk of serious complications.

Over the past two decades, a few less invasive methods, among others, pipelle, tao brush, vabra, jet irrigation, have been introduced to increase the availability, reduce the number and incidence of complications as well as lower the costs endometrial pathology diagnostics [6-9]. Pipelle has become 
very popular due to its easiness and simplicity as well as its high sensitivity in detecting endometrial cancer [10-12].

The aim of this study is to assess the effectiveness of pipelle in sampling diagnostic material from the uterine cavity and determining clinical factors which may affect its effectiveness.

\section{MATERIAL AND METHODS}

The retrospective analysis included 312 patients who underwent pipelle biopsy because of various indications in the $1^{\text {st }}$ Chair and Department of Obstetrics and Gynecology, Medical University of Warsaw, in the years 2013-2014. The study assessed the effectiveness of endometrial aspiration biopsy in sampling tissue material from the uterine cavity for histopathologic examination. To determine factors which may affect the effectiveness of this method the analysis covered: types of indications for the procedure, age, BMI, anteflexion or retroflexion of the uterus, presence of uterine fibroids, miscarriages, natural deliveries, deliveries in general.

The statistical analysis of the material was performed with the use of Statistica 12.0. In the analysis of measurable data (age, BMI), student t-test was used and, in the absence of normality, checked with Shapiro-WilkW test, the Mann-Whitney $U$ test was used. To evaluate the dependence of qualitative variables (shape of the uterus, presence of uterine fibroids, past uterine operations as well as procedures in the uterine cavity, miscarriages, natural deliveries, deliveries in general) chi-square test was used. The significance level adopted in all analyses was 0.05 .

\section{RESULTS}

Abnormal uterine bleeding was the most common indication for the procedure - 151 (48.4\%). In 161 (51.6\%) of cases indications other than abnormal uterine bleeding were present. Detailed distribution of indications is given in Figure 1.

In 259 (83.01\%) out of the 312 women in whom pipella was applied the final sampled material allowed for a histopathological diagnosis. Normal endometrium was found in 237 (75.96\%) of patients, in 17 (6.56\%) of cases endometrial hyperplasia was diagnosed and in 3 (0.96) endometrial cancer. In 53 (16.99\%) of the women we failed to sample from the uterine cavity tissue material which would allow to make a diagnosis on histopathological examination. Detailed figures are presented in Figure 2.

Among the analysed factors of potential impact on the diagnostic effectiveness of pipelle the following were found to be of significance: indications for the procedure, BMI, age and menopausal status $(p<0.05)$ (Table 1).

The assessment of the influence of indications for the procedure on the diagnostic effectiveness of pipelle after sampling tissue material gave the following results. In the group of patients with abnormal uterine bleeding diagnostic material was obtained in 133/151 (88.08\%) while in the case of biopsies performed for indications other than uterine bleeding material for microscopic examination was obtained in 126/151 (78.26\%) of patients which constituted a significantly lower figure $(p<0.05)$.

The analysis of the influence of BMI on the effectiveness of pipelle allowed to distinguish three prognostic groups. Most frequently diagnostic material was obtained in women with a BMI not exceeding 23. In this group adequate material was sampled in $79 / 87(90.8 \%)$ of patients. In the group with a BMI of $23-28,70 / 87$ (80.46\% diagnostic biopsies were recorded. The lowest percentage of positive results was found in the group of women with a BMI $>28$ where it

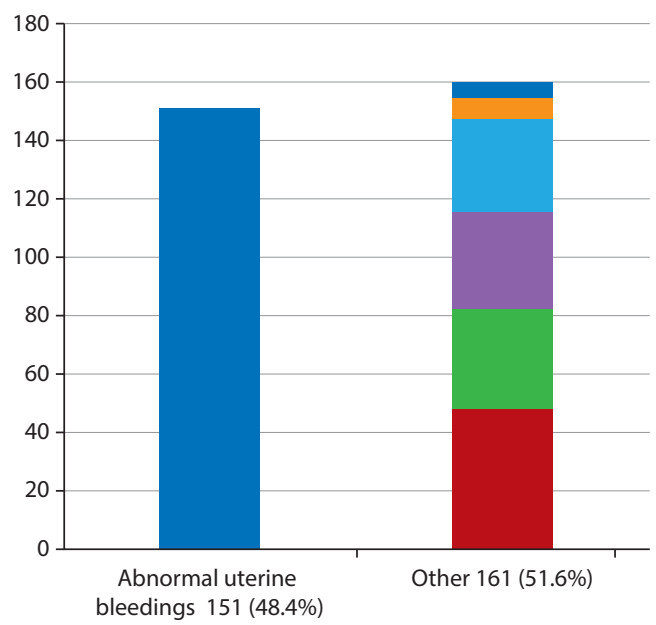

Diagnostics of spontaneous abortions, infertility irregular menstruation - 5 (3.11\%)

Pre-operative examination - 8 (4.97\%)

Cervical dysplasia - 32 (19.88\%)

State after treatment of simple hyperplasia - 33 (20.47\%)

Cervical polyps - 34 (21.12\%)

Abnormal USG picture of endometrium - 48 (29.81\%)

Column 1

Figure 1. Indications for endometrial aspiration biopsy with the use of pipelle. The most common indication for the performance of pipelle was abnormal uterine bleeding - 151 (48.4\%). Other indications for the procedure included: abnormal USG image - 48 (15.38\%), cervical polyp - 34 (10.9\%), state after simple hyperplasia - 33 (10.58\%), cervical dysplasia - 32 (10.26\%), pre-operative examination - $8(2.56 \%)$ and diagnostics of abortions, infertility, irregular menstruations $-5(1.6 \%)$ 


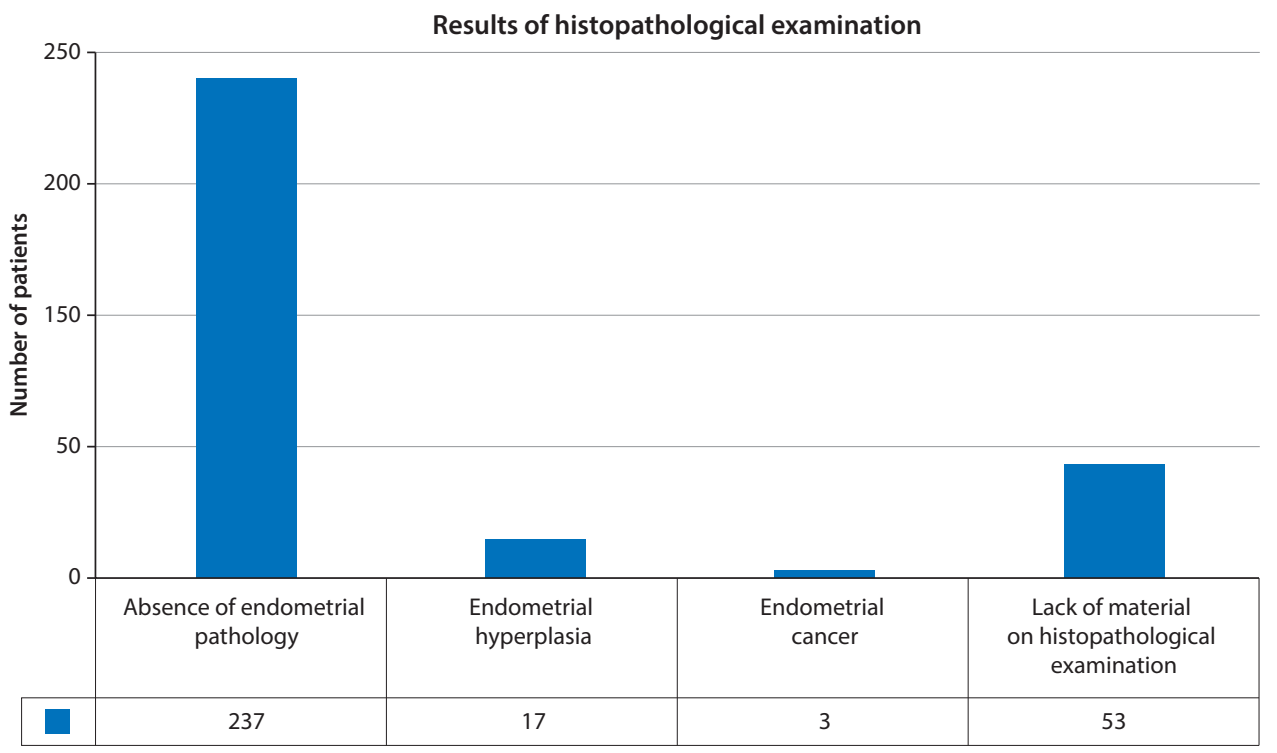

Figure 2. Distribution of results of histopathological examination obtained with the use of pipelle. In 23 cases no endometrial pathology was found, in 17 endometrial hyperplasia was diagnosed and in 3 endometrial cancer was detected. In 53 women no tissue material from uterine cavity was obtained

\begin{tabular}{|c|c|c|c|}
\hline \multicolumn{2}{|c|}{ Factors affecting the effectiveness of pipelle biopsy } & \multirow{3}{*}{$\begin{array}{c}\begin{array}{c}\text { Percentage } \\
\text { of non-diagnostic biopsies (\%) }\end{array} \\
111.51 \\
21.74\end{array}$} & \multirow{3}{*}{$\begin{array}{c}\text { Chi-square test } \\
\qquad P<0.05\end{array}$} \\
\hline \multirow{2}{*}{ Type of indication for the procedure } & Abnormal uterine bleeding & & \\
\hline & Other & & \\
\hline \multirow{3}{*}{ BMI } & $<23$ & 9.19 & \\
\hline & $23-28$ & 19.5 & $P<0.05$ \\
\hline & $>28$ & 23.17 & \\
\hline \multirow{3}{*}{ Age } & $<45$ yrs. & 3.85 & \\
\hline & $45-55$ yrs. & 14.95 & $P<0.001$ \\
\hline & $>55$ yrs. & 41.33 & \\
\hline \multirow{3}{*}{ Menopausal status } & Menstruating & 5.18 & \\
\hline & Peri-menopausal & 29.63 & $P<0.001$ \\
\hline & Post-menopausal & 38.46 & \\
\hline
\end{tabular}

reached $63 / 82$ (76.83\%). The differences between the groups were statistically significant at $\mathrm{p}<0.05$.

The age criterion allowed to distinguish three groups of patients, the first being women $<45$ years of age, the second women between 45 and 55 years of age and the third women $>55$ years of age. The effectiveness of biopsy performed with the use of the pipelle device was the highest in the group of patients over 45 years of age where material for microscopic examination was obtained from $125 / 130$ (96.15\%) of the women. In older patients, the effectiveness of this method of sampling biopsy material was lower. In the 45-55 age group diagnostic biopsies were successfully performed in 91/107 (85.05\%) of patients while in the $>55$ age group in 44/75 (58.67\%) of women. The differences observed between the groups were statistically significant $(p<0.05)$.

The menopausal status of the women was another parameter analysed. Diagnostic biopsies were most common in the pre-menopausal group of patients where tissue material from the uterine cavity was obtained in 183/193 (94.82\%) women. In peri-menopausal and post-menopausal patients the effectiveness was lower, diagnostic biopsies being obtained in 19/27 (70.37\%) and 56/91 (61.54\%), respectively $(p<0.05)$.

The study did not reveal the influence of other factors on the effectiveness of pipelle in sampling tissue material 
for histopathological examination. Anteflexion of the uterus was found to be associated with a slightly higher percentage of effective biopsies than retroflexion ( $84.94 \%$ vs. $80.49 \%$, $p=0.61$ ). The presence of fibroids in the uterus can have an adverse impact on sampling material from the uterine cavity (79.55\% vs. $83.96 \%)$, however this does not constitute a statistically significant difference $(p=0.47)$. Past history of operations on the uterus or procedures within the uterine cavity was of no impact on sampling material from the cavity, $80.43 \%$ vs. $83.52 \%$ ( $p=0.6$ ) and $84.03 \%$ vs. $79.59 \%$ $(p=0.44)$. In nulliparas, the percentage of diagnostic biopsies was higher but not in a statistically significant way $(89.29 \%$ vs. $82.03 \%, p=0.19)$.

\section{DISCUSSION}

Early detection of pre-neoplastic and neoplastic changes remains the principal aim of endometrial diagnostics. Less invasive techniques which can be applied in an outpatient setting play a primary role these days. Pipelle endometrial biopsy seems to enjoy the greatest popularity among them [10-12]. Though a mere $4.2 \%$ of the uterine cavity surface can be inspected during its performance, the sensitivity and specificity of pipelle in detecting hyperplasia and atypia are comparable with abrasion and reach $67 \%$ and $75 \%$, respectively $[10,13]$. In the case of abrasion, they are $62 \%$ and $83 \%$, respectively [13]. Pipelle biopsy is also characterized by high sensitivity in detecting endometrial neoplasms (93.8-99.2\%) [14]. Lack of need for general anaesthesia, reduced duration of the diagnostic procedure, cost reduction and decrease in the number and incidence of complications constitute no doubt further significant advantages of this diagnostic method [15].

The way in which material is sampled from the uterine cavity with the use of this method may reduce its effectiveness in obtaining a reliable quantity of tissue material for microscopic examination. Different authors report a percentage of non-diagnostic examinations of between 3 and $16 \%$ $[13,16-18]$. In their meta-analysis Dijkuzen et al. estimated that $10.4 \%$ of pipelle biopsies involve ineffective sampling of uterine cavity material [4]. In our study, the percentage of ineffective pipelle biopsies was $16.99 \%$.

An additional aim of the study was to determine factors which may have an influence on the effectiveness of pipelle. Like in the study by Williams et al. important factors proved to be the age of patients and their menopausal status strictly linked to it [19]. In women with atrophic endometrium the thickness of which on USG examination was $<5 \mathrm{~mm}$ the effectiveness of pipelle biopsy in obtaining adequate material from the uterine cavity is merely $27 \%$ [20]. On the other hand, Williams et al. report that the percentage of non-diagnostic results in women with AUB after menopause was $48 \%$ while in women over 54 years of age it is even higher and reaches 58\% [19]. In our study, the diagnostic effectiveness of pipelle biopsy was also the lowest in post-menopausal women and in the age group $>55$ years of age, reaching $38.46 \%$ and $41.33 \%$, respectively. The differences in the obtained results can be explained with differences in the population groups studied. We analysed pipelle biopsies performed for all indications, not only for abnormal uterine bleeding.

It seems that when the indication for biopsy is other than abnormal uterine bleeding, the use of another diagnostic method may be a better choice. However, due to the high diversity of our study group (as many as 6 different indications - abnormal USG image of the endometrium, polyp of the cervix, state after treatment of endometrial hyperplasia, cervical dysplasia, diagnostics prior to a planned surgical procedure, spontaneous abortion diagnostics, infertility, irregular menstruation) we find it difficult to point out in what types of indications pipelle biopsy is clearly a bad choice as a method of first choice and where it may be considered. What arouses our greatest doubts is the performance of pipelle biopsy as a check-up after treatment of endometrial hyperplasia, in particular with atypia. Failure to obtain diagnostic material may then become a cause of unnecessary stress and uncertainty for women and of delays in further management where treatment proves ineffective.

In our study, we showed that performance of endometrial aspiration biopsy for indications other than AUB is linked to a higher percentage of non-diagnostic biopsies ( $21.74 \%$ vs. $11.51 \%)$. In the studies referred to, their authors investigated the effectiveness of pipelle biopsy but in their analyses they did not take into account the influence of individual types of indications on the effectiveness of the method. The majority of the studies concerns pipelle biopsies performed because of abnormal uterine bleeding. Korean authors assessed the effectiveness of sampling material from the uterine cavity in which LNG-IUS was placed. They showed that inadequate material was harvested in as much as $60.7 \%$ [19]. What attracts attention is the heterogeneity of the study group with as many as 8 types of indications (abnormal USG image of the endometrium, polyp of the uterine cervix, state after endometrial hyperplasia, dysplasia of the uterine cervix, diagnostics prior to a planned surgical procedure, diagnostics of spontaneous abortions, infertility, irregular menstruations). Further studies on larger groups of women are needed to determine which of the indications listed are of major impact on the diagnostic effectiveness of pipelle biopsy.

A factor of significant consequence for the diagnostic effectiveness of pipelle biopsy, not described in earlier studies, is BMI. In our study, we registered the highest percentage of 
failed biopsies in obese and overweight women (BMI > 28). This can be explained in terms of technical difficulties with performing the procedure. However, we found significantly worse effectiveness in women with BMI 23-28 while obtaining the best effectiveness in the group of patients with $\mathrm{BMI}<23$. This factor requires being checked in more detail in subsequent studies. Importantly, in none of the articles referred to was this parameter studied as a factor of potential influence on the effectiveness of pipelle biopsy. BMI is a parameter easy to calculate in everyday clinical practice and its inclusion can increase the percentage of results of no diagnostic value while at the same time eliminate the necessity of repeating the examination in the case of failure to obtain material for microscopic examination.

Williams et al. report nulliparity being a factor of adverse effect for the diagnostic effectiveness of pipelle biopsy [19]. Among women who gave birth to a child the percentage of results of no diagnostic value was $5 \%$ while in the group of nulliparas it reached $25 \%$. These results were independent of the ability to enter the uterine cavity as well as statistically significant $(p<0.001)$. Our findings do not confirm this dependence. The percentage of non-diagnostic results in nulliparas was $10.71 \%$ (6/56 of cases). On the other hand, in child-bearing women it was $17.9 \%$ (46/256 of cases). However this difference was not statistically significant $(p=0.18)$.

The principal aim of endometrial diagnostics is to detect a malicious neoplasm and pathologic hyperplasia. Over the past years we have been observing growth in the incidence of endometrial cancer among women of post-menopausal age $[21,22]$. So far no screening method which would help detect early forms of this neoplasm has been proved to exist. This is precisely what makes it extremely important to have a possibility to apply an effective diagnostic method in the case of the presence of risk factors or on the appearance of disquieting symptoms. It turns out that otherwise commonly known endometrial cancer risk factors such as obesity, advanced age or menopausal status can also be factors of negative influence on the diagnostic effectiveness of pipelle biopsy. In these cases, curettage of the uterine cavity seems to be able to accelerate diagnosis and thus treatment. On the other hand, patients with endometrial cancer risk factors are women with numerous internal medicine comorbidities, requiring numerous consultations, additional examinations and time for adequate preparation for general anaesthesia and abrasion. In cases of this kind, pipelle biopsy can constitute a method of first choice, in particular when the time of waiting for the result of a histopathological examination is several days.

All methods of the endometrial biopsy are related to the possibility of various complications. Pain is the most commonly reported by women undergoing aspiration biopsy. It has been demonstrated that pipelle endometrial sampling compared to $D \& C$ biopsy is associated with less pain [10, 15]. Other study concluded that pipelle significantly produced more pain than D \& C and higher overall discomfort [23]. It has been shown that the frequency and severity of pain is closely associated with the use tenaculum during inserting a pipelle into the uterine cavity. Using the tenaculum was found to increase the risk of painful biopsy 32-fold [24]. Therefore it is recommended not to use tenaculum, unless it is necessary for the biopsy.

The highest risk of serious complications occurs at $D \& C$ biopsy and it is estimated at $0.6-1.3 \%$ for perforation, $0.3-$ $-0.5 \%$ for infections, $0.4 \%$ for bleeding/haemorrhage $[25,26]$. Endometrial sampling with pipelle device reduced incidence of serious complications [27]. Although our study did not report any uterine muscle damage, pelvic infection or other complications, it is estimated that the risk of perforation during pipelle biopsy is $0.1-0.2 \%$ [28]. One of the most severe complication was the case of large pelvic abscess following uterine perforation in a 53-year-old woman after endometrial pipelle sampling [29]. Loops of the large bowel were encased in the abscess. Despite the use a broad spectrum antibiotics (ampicillin, gentamicin, clindamycin) no improvement of the general condition has been reported. Women required surgical treatment - modified radical hysterectomy, bilateral adnexectomy, lysis of adhesions and partil colon resection with end-to-end anastomosis were performed. Although pipelle endometrial sampling is a minimally invasive procedure, clinicians always should be aware of the existing risks of serious complications.

Endometrial aspiration biopsy with the use of pipelle is a diagnostic method alternative to abrasion. The development of the outpatient diagnostics of endometrial pathologies increases the availability of this examination to the population at large, shortens the time and is evidently cost-effective. Knowledge of factors of potential influence on the effectiveness of pipelle biopsy in obtaining diagnostic material can make proper qualification easier for gynecologists.

We would recommend to commence diagnostics with pipelle biopsy in women $<45$ years of age, with abnormal uterine bleeding, before menopause and with normal BMI. On the other hand, in asymptomatic patients, obese, elder and non-menstruating women it seems better to begin the diagnostic procedure with a method other than pipelle biopsy.

A larger number of studies on factors of potential influence on the effectiveness of pipelle biopsy is obviously necessary to determine beyond any doubt which diagnostic method would constitute the best choice for a given patient. In particular, this concerns factors for which statistical significance was not assessed. 


\section{CONCLUSIONS}

The diagnostic effectiveness of pipelle in sampling diagnostic material depends on the types of indications for the procedure, BMI, age and menopausal status. Its diagnostic value can decrease significantly when a few adverse factors overlap.

The diagnostic value of pipelle was found to be the lowest in women with overweight and obesity (BMI > 28), post-menopausal women, women over 55 years of age and where biopsy was performed for indications other than abnormal uterine bleeding.

On the other hand, pipelle shows clearly the highest effectiveness in sampling diagnostic material from the uterine cavity in young women ( $<45$ years of age), menstruating women, women with normal body weight or underweight $(\mathrm{BMI}<23)$ and abnormal uterine bleeding.

We can also distinguish a group of women in whom pipelle shows indirect effectiveness in sampling tissue material, namely, patients aged 45 to 55 years, in peri-menopausal period, with a BMI coefficient of 23-28.

\section{REFERENCES}

1. MC, Le T, Le T, [et al.]. SOGC-GOC-SCC Policy and Practice Guidelines Committee. Epidemiology and investigations for suspected endometrial cancer. J Obstet Gynaecol Can. 2013, 35 (4), 380-383.

2. Wamsteker K. Hysteroscopy in the management of abnormal uterine bleeding in 199 patients. In: Seigler AM, Lindemann HJ, eds. Hysteroscopy: principles and practice. $1^{\text {st }}$ ed. Philadelphia, JB Lippincott 1984, 128-131.

3. Stock RJ, Kanbour A. Prehysterectomy curettage. Obstet Gynecol. 1975, 45, 537-541.

4. Dijkhuizen FP, Mol BW, Brölmann HA, Heintz AP. The accuracy of endometrial sampling in the diagnosis of patients with endometrial carcinoma and hyperplasia: a meta-analysis. Cancer. 2000, 89 (8), 1765-1772.

5. Guido RS, Kanbour-Shakir A, Rulin MC, Christopherson WA. Pipelle endometrial sampling. Sensitivity in the detection of endometrial cancer. J Reprod Med. 1995, 40 (8), 553-555.

6. Maksem JA. Performance characteristics of the Indiana University Medical Center endometrial sampler (Tao Brush) in an outpatient office setting, first year's outcomes: recognizing histological patterns in cytology preparations of endometrial brushings. Diagn Cytopathol. 2000, 22 (3), 186-195.

7. Goldberg GL, Tsalacopoulos G, Davey DA. A comparison of endometrial sampling with the Accurette and Vabra aspirator and uterine curettage. SAfr Med J. 1982, 61 (4), 114-116.

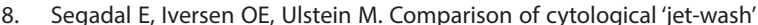
specimens and histology in endometrial carcinoma. J Clin Pathol. 1980, 33 (7), 688-690.

9. Bakkum-Gamez JN, Gonzalez-Bosquet J, Laack NN, Mariani A, Dowdy SC. Current issues in the management of endometrial cancer. Mayo Clin Proc. 2008, 83 (1), 97-112. doi: 10.4065/83.1.97.

10. Rauf R, Shaheen A, Sadia S, [et al.]. Outpatient endometrial biopsy with Pipelle vs diagnostic dilatation and curettage. J Ayub Med Coll Abbottabad. 2014, 26 (2), 145-148.
11. Leitao MM Jr, Kehoe S, Barakat RR, [et al.]. Comparison of D \& C and office endometrial biopsy accuracy in patients with FIGO grade 1 endometrial adenocarcinoma. Gynecol Oncol. 2009, 113 (1), 105-108.

12. Huang GS, Gebb JS, Einstein MH, Shahabi S, Novetsky AP, Goldberg $\mathrm{GL}$. Accuracy of preoperative endometrial sampling for the detection of high-grade endometrial tumors. Am J Obstet Gynecol. 2007, 196 (3), 243.e1-5.

13. Demirkiran F, Yavuz E, Erenel H, Bese T, Arvas M, Sanioglu C. Which is the best technique for endometrial sampling? Aspiration (pipelle) versus dilatation and curettage (D \& C). Arch Gynecol Obstet. 2012, 286 (5), 1277-1282.

14. Sanam M, Majid MM. Comparison the diagnostic value of dilatation and curettage versus endometrial biopsy by Pipelle - a clinical trial. Asian Pac J Cancer Prev. 2015, 16 (12), 4971-4975.

15. Liu H, Wang FL, Zhao YM, Yao YQ, Li YL. Comparison of Pipelle sampler with conventional dilatation and curettage (D \& C) for Chinese endometrial biopsy. J Obstet Gynaecol. 2015, 35 (5), 508-511.

16. Machado F, Moreno J, Carazo M, León J, Fiol G, Serna R. Accuracy of endometrial biopsy with the Cornier pipelle for diagnosis of endometrial cancer and atypical hyperplasia. Eur J Gynaecol Oncol. 2003, 24 (3-4), 279-281.

17. Elsandabesee D, Greenwood P. The performance of Pipelle endometrial sampling in a dedicated postmenopausal bleeding clinic. J Obstet Gynaecol. 2005, 25 (1), 32-34.

18. Williams AR, Brechin S, Porter AJ, Warner P, Critchley HO. Factors affecting adequacy of Pipelle and Tao Brush endometrial sampling. BJOG. 2008, 115 (8), 1028-1036.

19. Bray F, Dos Santos Silva I, Moller H, Weiderpass E. Endometrial cancer incidence trends in Europe: underlying determinants and prospects for prevention. Cancer Epidemiol Biomarkers Prev. 2005, 14 (5), 1132-1142.

20. Kim MK, Seong SJ, Song T, [et al.]. Comparison of dilatation \& curettage and endometrial aspiration biopsy accuracy in patients treated with high-dose oral progestin plus levonorgestrel intrauterine system for early-stage endometrial cancer. Gynecol Oncol. 2013, 130 (3), 470-473.

21. Münstedt K, Grant Ph, Woenckhaus J, [et al.]. Cancer of the endometrium: current aspects of diagnostics and treatment. World J Surg Oncol. 2004, 2, 24.

22. Jamison PM, Noone AM, Ries LA, [et al.]. Trends in endometrial cancer incidence by race and histology with a correction for the prevalence of hysterectomy, SEER 1992 to 2008. Cancer Epidemiol Biomarkers Prev. 2013, 22, 233.

23. Rezk M, Sayyed T, Dawood R. The effectiveness and acceptability of Pipelle endometrial sampling versus classical dilatation and curettage: a three-year observational study. Gynecol Obstet Invest. 2016. Doi: $10.1159 / 000444711$.

24. Kucukgoz Gulec U, Khatib G, Guzel AB, [et al.]. The necessity of using tenaculum for endometrial sampling procedure with pipelle: a randomized controlled study. Arch Gynecol Obstet. 2014, 289 (2), 349-356.

25. Grimes DA. Diagnostic dilation and curettage: a reappraisal. Am J Obstet Gynecol. 1982, 142 (1), 1-6.

26. Seamark CJ. The demise of the D \& C. JR Soc Med. 1998, 91 (2), 76-79.

27. Akhtar AZ. Pipelle, an acceptable ont patient technique for endometrial biopsy. JCPSP. 1999, 9, 14-16.

28. Cooper JM, Erickson ML. Endometrial sampling techniques in the diagnosis of abnormal uterine bleeding. Obstet Gynecol Clin North Am. 2000, 27, 235.

29. Abulafia O, Shah T, Salame G, [et al.]. Sonographic and magnetic resonance imaging findings of pelvic abscess following uterine perforation sustained during office endometrial sampling. J Clin Ultrasound. 2011, $39(5), 283-286$ 